YITP-17-03, IPMU17-0011

\title{
CMB Scale Dependent Non-Gaussianity from Massive Gravity during Inflation
}

\author{
Guillem Domènech ${ }^{1}$, Takashi Hiramatsu ${ }^{1,2}$, Chunshan $\operatorname{Lin}^{1}$, Misao Sasaki ${ }^{1}$, \\ Maresuke Shiraishi ${ }^{3}$, Yi Wang ${ }^{4}$ \\ 1 Center for Gravitational Physics, Yukawa Institute for Theoretical Physics, Kyoto University, \\ 606-8502, Japan \\ 2 Department of Physics, Rikkyo University, Toshima, Tokyo, 171-8501, Japan \\ ${ }^{3}$ Kavli Institute for the Physics and Mathematics of the Universe (Kavli IPMU, WPI), \\ UTIAS, The University of Tokyo, Chiba, 277-8583, Japan \\ ${ }^{4}$ Department of Physics, The Hong Kong University of Science and Technology, \\ Clear Water Bay, Kowloon, Hong Kong, P.R.China
}

\begin{abstract}
We consider a cosmological model in which the tensor mode becomes massive during inflation, and study the Cosmic Microwave Background (CMB) temperature and polarization bispectra arising from the mixing between the scalar mode and the massive tensor mode during inflation. The model assumes the existence of a preferred spatial frame during inflation. The local Lorentz invariance is already broken in cosmology due to the existence of a preferred rest frame. The existence of a preferred spatial frame further breaks the remaining local $\mathrm{SO}(3)$ invariance and in particular gives rise to a mass in the tensor mode. At linear perturbation level, we minimize our model so that the vector mode remains non-dynamical, while the scalar mode is the same as the one in single-field slow-roll inflation. At non-linear perturbation level, this inflationary massive graviton phase leads to a sizeable scalar-scalar-tensor coupling, much greater than the scalar-scalar-scalar one, as opposed to the conventional case. This scalar-scalar-tensor interaction imprints a scale dependent feature in the CMB temperature and polarization bispectra. Very intriguingly, we find a surprizing similarity between the predicted scale dependence and the scale-dependent non-Gaussianities at low multipoles hinted in the WMAP and Planck results.
\end{abstract}




\section{Contents}

1 Introduction 2

2 Spacetime Symmetry Breaking during Inflation 3

3 Primordial Perturbation Spectra $\quad 6$

3.1 Linear Perturbation $\quad 6$

$\begin{array}{llr}3.2 & \text { Non-linear Perturbations } & 9\end{array}$

3.3 Primordial 3-point Correlation Function 10

4 CMB Bispectra $r$

5 Conclusion and Discussion $r$

$\begin{array}{lr}\text { A On the gauges } & 17\end{array}$ 


\section{Introduction}

Inflation is the leading paradigm of the very early universe cosmology [1-4]. It successfully explains the CMB temperature fluctuation and large scale structure that we observe nowadays. Despite the great success of inflationary paradigm, its origin still remains unknown. One of the simplest theoretical possibilities is that inflation was driven by a single scalar field, called inflaton, rolling on a slightly tilted platform of its potential. In the regime where the inflaton moves sufficiently slowly and monotonically, there is a one-to-one correspondence between the value of the inflaton and the cosmic time, giving rise to a preferred time slicing defined by the dynamics of the inflaton.

Recently, there has been a growing interest in the inflationary physics with broken spatial reparameterisation [5-12]. From the general relativistic point of view, one introduces 3 scalar fields which determine a preferred spatial frame. The configuration of the 3 scalar fields is such that the energy momentum tensor is homogeneous and isotropic on the background but ceases to be so at the perturbation level. This induces an effective mass to the transverse and traceless part of the spatial metric, i.e. the tensor mode (which is eventually identified with the graviton). In particle physics language, there appear 3 Nambu-Goldstone bosons associated with the broken spatial re-parameterisation invariance (4 in total if we include the one associated with broken time re-parameterisation invariance due to the inflaton dynamics). In the unitary gauge, these 3 Goldstone bosons are eaten by one of the scalar degrees and two degrees of the tensor mode in the spatial metric. Thus in particular the tensor mode becomes massive. In this sense, general relativity is extended to a theory with non-vanishing graviton mass.

Searching for a finite range gravity is a basic question of classical field theory. It can be traced all the way back to the pioneering work by Fierz and Pauli in 1939 [13]. See Ref. [14-24] for some relevant references and Ref. [25] for a comprehensive review on the recent progresses in this topic. Besides the motivation from a purely theoretical interest, the question whether gravity needs to be modified in the very early universe is particularly important due to the Lyth bound [26]. According to it, the tensor-to-scalar ratio $r$ is proportional to the variation of the inflaton field during inflation. It can be quite generically shown that the amplitude of the primordial gravitational waves with $r \gtrsim 10^{-3}$ requires a super-Planckian excursion of the inflaton. which is generally regarded as being out of the validity of the low energy effective field theory. In the near future, several next-generation satellite missions as well as the ground based and balloons experiments, are aimed at measuring primordial gravitational waves down to $r \sim 10^{-3}$ [27-29]. Thus, the detection of a primordial tensor perturbation with sufficiently large amplitude has a profound impact on our understanding of fundamental physics. It implies either gravity or quantum field theory needs to be modified in the very early universe. See [11] for example for a mechanism to evade the Lyth bound by means of a parametric resonance in the context of massive gravity.

Another key focus in modern cosmology is non-Gaussianities in the CMB fluctuations. WMAP reported a hint of a local-type non-Gaussianity, but the confidence level was smaller than $2 \sigma$ and thus nothing firm was concluded [30,31]. The Planck satellite improved the constraints on non-Gaussianities with high multipole $\ell$ data with better precision $[32,33]$. The current constraint on the scale independent local-type non-Gaussianity is $f_{N L}^{\text {local }}=2.5 \pm 5.7$ [33]. Interestingly enough, if the maximum multipole moment is taken to be comparable to that of WMAP (around $\ell_{\max }=500$ ), the Planck result also indi-

cates a positive local non-Gaussianity, while for $\ell_{\max }>1000, f_{N L}^{\text {local }}$ found to be vanishingly small. This suggests a scale dependent local non-Gaussianity. The bispectrum for the E-mode polarization shows a very similar pattern as well, providing an additional support for such scale dependence. 
Assuming that the scale dependent local non-Gaussianity is of primordial origin, there can be roughly two straightforward explanations. The first possibility is to tune the scale dependence by controlling the parameters in multi-field inflation [34-38] or quasi-single field inflation [39-41] (where quasi-local-type non-Gaussianity can be generated). The first constraint on the running of non-Gaussianity of scalar perturbations can be found in the Ref. [42]. The second possibility is to generate the scale dependence through the scalar-scalar-tensor coupling. However, the tensor mode produces a much smaller amplitude compared to that induced by the scalar mode (recall that the tensor-to-scalar ratio is less than 0.1). Nevertheless, if the tensor mode were highly non-Gaussian, they could significantly contribute to nonGaussianities in the temperature and E-mode polarization maps. Some similar ideas have been pursued to generate low $\ell$ CMB anomalies such as power asymmetries [43] and quadrupolar anisotropy [44]. Resorting to the tensor mode for a scale-dependent non-Gaussianity has an advantage, compared to the multi-field or quasi-single field case, that one only needs to fit the amplitude since the scale dependence is naturally obtained by the decay of the tensor perturbations (gravitational waves) after they enter the horizon.

In this paper, we present a novel model where a sufficiently large scale-dependent local-type nonGaussianity is generated from the scalar-scalar-tensor coupling. This has not been realized before, to the best of our knowledge, simply due to the fact that, since $r<0.1$, all the inflationary models in the literature which generates a large tensor non-Gaussianity generates an even larger scalar non-Gaussianity. ${ }^{1}$ As a result, the scale-dependence of the non-Gaussianity due to the tensor mode is swamped by the scaleindependent non-Gaussianity from the scalar sector, and is constrained to be small by the Planck result on non-Gaussianities up to $\ell \sim 2000$.

In the current work, we show that with the help of spatial symmetry breaking, the tensor nonGaussianity can be much larger than the scalar non-Gaussianity. The paper is organized as follows. In Section 2, we build an inflationary model with spatial symmetry breaking. In Section 3, we calculate the primordial fluctuations of the model, including the scalar and tensor power spectra and the non-Gaussianities. In Section 4, we compute the CMB temperature and polarization bispectra from the primordial scalar-scalar-tensor coupling. We conclude in Section 5. Further details on the calculations are presented in Appendix.

\section{Spacetime Symmetry Breaking during Inflation}

In this section, we build our model by means of the low energy Effective Field Theory approach. We consider a spatially flat background with the 3-metric of the form,

$$
d s_{(3)}^{2}=a^{2} \delta_{a b} e_{i}^{(a)} e_{j}^{(b)} d x^{i} d x^{j}
$$

where $a$ is the cosmic scale factor, and $e_{i}^{(a)}$ is the triad basis representing the local $\mathrm{SO}(3)$ symmetry, which satisfies $\delta_{a b} e_{i}^{(a)} e_{j}^{(b)}=\delta_{i j}$, with $a, b$ being the internal indices and $i, j$ the space indices, On this background we consider a model in which there is a preferred spatial frame during inflation. Namely the local $\mathrm{SO}(3)$ symmetry is broken by the existence of a preferred, rigid spatial frame, say $e_{i}^{(a)}=\delta_{i}^{a}$.

A minimal description for this preferred spatial frame is to introduce 3 Stueckelberg scalar fields,

$$
\varphi^{a}=\delta_{i}^{a} x^{i}+\pi^{a}
$$

\footnotetext{
${ }^{1}$ See Ref. [45] for an inflationary model with an axion-like spectator field realizing an equilateral-type non-Gaussianity sourced mainly by the gravitational wave sector.
} 
where $\pi^{\prime} s$ are three Nambu-Goldstone bosons that nonlinearly recover the local $\mathrm{SO}(3)$ symmetry. The internal symmetries we impose on our theory are the $\mathrm{SO}(3)$ rotational symmetry and rescaling symmetry,

$$
\varphi^{a} \rightarrow \Lambda_{b}^{a} \varphi^{b}, \quad \varphi^{a} \rightarrow \lambda \varphi^{a} .
$$

In passing we mention that since there always exists a preferred time slicing or rest frame in cosmology, and the inflaton field $\phi$ determines the preferred rest frame during inflation, one may regard the set $\left(\phi, \varphi^{a}\right)$ as the 4 Stueckelberg scalar fields that recovers the local Lorentz (i.e. SO(3,1)) symmetry. However, below we will not take this view but only focus on the spatial symmetry breaking.

We follow the useful notation introduced in Ref. [5],

$$
Z^{a b} \equiv g^{\mu \nu} \partial_{\mu} \varphi^{a} \partial_{\nu} \varphi^{b}, \quad \bar{\delta} Z^{a b} \equiv \frac{Z^{a b}}{Z}-3 \frac{\delta_{c d} Z^{a c} Z^{b d}}{Z^{2}},
$$

where $Z$ is the trace of $Z^{a b}$. These nontrivial VEVs of the fields are the origin of a non-vanishing graviton mass. The action can be presumptively written as

$$
S=\int d^{4} x \sqrt{-g}\left[\frac{1}{2} M_{p}^{2} \mathcal{R}-\frac{1}{2} g^{\mu \nu} \partial_{\mu} \phi \partial_{\nu} \phi-V(\phi)-\frac{9}{8} M_{p}^{2} m_{g}^{2}\left(\bar{\delta} Z^{a b}\right)^{2}+\cdots\right]
$$

where $m_{g}^{2}$ is the graviton mass, $\left(\bar{\delta} Z^{a b}\right)^{2}=\delta_{a c} \delta_{b d} \bar{\delta} Z^{a b} \bar{\delta} Z^{c d}$, and the last dots stand for the higher order operators which only appear at nonlinear perturbation level. We assume a functional dependence of $m_{g}^{2}$ on another scalar field, e.g. inflaton, so that during inflation the graviton mass scale is around the typical scale of inflation, and after inflation the graviton mass vanishes or reduce to a very small value which is below current observational bound [46]. Note that $\bar{\delta} Z^{a b}$ is defined in the way that it does not contribute to the background energy momentum tensor, and it is traceless at linear perturbation level. Thus, the background dynamics is totally determined by the slow rolling of the inflaton scalar field.

According to the Nambu-Goldstone theorem, one may also expect 3 additional Nambu-Goldstone bosons to appear in the inflationary perturbation spectra, in addition to the scalar degree associated with the inflaton scalar. In the unitary gauge, in which we set $\pi^{a}=0$, one would also expect these 3 Nambu-Goldstone bosons are eaten by the degrees of freedom in the spatial metric, which we call the graviton for simplicity, and the graviton develops one helicity 0 and two helicity 1 modes. However, this is not always necessarily the case due to the Lorentz symmetry breaking (i.e. the existence of the preferred rest frame) of the background. In fact these 3 degrees of $\pi^{\prime} s$ turn out to be non-dynamical at leading order in gradient expansion, in other words, at tree level in the context of quantum field theory.

The reason is because of the $S O(3)$ rotational symmetry and the rescaling symmetry given by Eq. (2.3). Consider a perturbation $\pi^{a}$ in the long wavelength limit. In this limit, the general form of $\pi^{a}$ will be

$$
\pi^{a}=\rho(x) \delta_{j}^{a} x^{j}+\omega^{a b}(x) \delta_{b j} x^{j},
$$

where $\omega^{a b}$ is antisymmetric in its indices and the spatial derivatives of $\rho$ and $\omega^{a b}$ are assumed to negligible in comparison with the Hubble scale, namely,

$$
\partial_{i} \rho \ll H \rho, \quad \partial_{i} \omega^{a b} \ll H \omega^{a b} .
$$

The term proportional to $\rho$ in Eq. (2.6) corresponds to the helicity 0 mode (scalar-type perturbation), and the one proportional to $\omega^{a b}$ to the helicity 1 modes (vector-type perturbation). It is then easy to see 
that these two types of perturbations just reduce to an infinitesimal symmetry transformation (2.3) at leading order in gradient expansion. Hence they cannot be dynamical.

In what follows, for the sake of notational simplicity, we identify the internal indices $a, b, \cdots$ with the space indices $i, j, \cdots$, which actually corresponds to the particular choice of the spatial frame $e_{i}^{(a)}=\delta_{i}^{a}$, and also omit the kronecker delta in summation over the indices, e.g. $\delta_{i j} \pi^{i} \pi^{j} \rightarrow \pi^{i} \pi^{i}$, unless there is a chance of confusion.

In the decoupling limit, the quadratic action of Nambu-Goldstone bosons are calculated as

$$
\begin{aligned}
S_{\pi} & =\frac{9}{4} \Lambda^{4} \int \partial_{i} \pi^{j} \partial_{i} \pi^{j}+\frac{1}{3}\left(\partial_{i} \pi^{a}\right)^{2} \\
& \sim \Lambda^{4} k^{2} \pi^{i} \pi^{i},
\end{aligned}
$$

where $\Lambda^{2} \equiv M_{p} m_{g}$ is the UV cut-off of our theory. The absence of the kinetic term at the level of lowest dimensional operator implies that these $3 \mathrm{Nambu-Goldstone} \mathrm{bosons} \mathrm{require} \mathrm{the} \mathrm{kinetic} \mathrm{term} \mathrm{from} \mathrm{higher}$ dimensional operators. For instance, if we include the term $g^{\mu \nu} \partial_{\mu} \bar{\delta} Z^{i j} \partial_{\nu} \bar{\delta} Z^{i j}$, the Nambu-Goldstone boson action schematically reads

$$
S_{\pi}=\Lambda^{4} k^{2}\left(\pi^{i}\right)^{2}+\Lambda^{2} k^{2}\left(\dot{\pi}^{i}\right)^{2}
$$

After canonical normalisation $\pi^{i} \rightarrow \Lambda k \pi^{i}$, we found these 3 Nambu-Goldstone bosons become supermassive $m_{\pi}^{2} \sim \Lambda^{2}$. During inflation, the energy scale of the mixing between the inflaton and gravity is characterised by $\Lambda_{m i x}^{2} \sim \dot{H}$. In a massive gauge field theory, the mixing scale is generally characterised by the mass scale of the gauge field. If we assume that the massive gravity and inflation are governed by the same physics, it is natural to identify the graviton mass with the mixing scale, namely we have $m_{g}^{2} \sim \dot{H}$. Then the mass of these 3 Nambu-Goldstone bosons will be given by $m_{\pi}^{2} \sim M_{p} \dot{H}^{1 / 2} \gg H^{2}$. Thus any excitations of these bosons exponentially decay away during inflation, and their existence becomes completely ignorable.

Assuming the above argument is more or less valid, we will simply neglect these 3 Nambu-Goldstone pions. Then considering these 3 modes in the unitary gauge, it is apparent that the scalar and vector type perturbations corresponding to these 3 modes remain non-dynamical. Namely, the momentum constraints still kill the 3 degrees of freedom. Meanwhile, as we will see in the section 3.1, the tensor mode (gravitational waves) receives a modest mass correction $m_{G W}^{2} \sim m_{g}^{2} \ll H^{2}$. The broken Lorentz invariance, as well as the remaining symmetry of the scalar field configuration (2.3) is the origin of this mass hierarchy.

Therefore, at low energy scale, our model has only 3 degrees of freedom; one scalar mode associated with the fluctuations of the inflaton field and two massive tensor modes. We will confirm this point in the following detailed perturbation analysis. In the long wavelength limit $k \rightarrow 0$, one may worry that the kinetic term vanishes again and our theory becomes strongly coupled. However, a small but non-vanishing kinetic term of Nambu-Goldstone pion can always arise from loop corrections or global symmetry $\varphi^{i} \rightarrow \lambda \varphi^{i}$ breaking at ultra-low energy scale, and protects our theory from the strong coupling problem.

In principle, there are also possible problematic terms such as $\left(\partial^{2} \pi^{a}\right)^{2}$ at the level of higher order derivatives. The health of our theory requires that these terms should appear in the Galileon form [47],

$$
\nabla^{2} \pi^{a} \nabla^{2} \pi^{b}-\left(\nabla_{\mu} \nabla_{\nu} \pi^{a}\right)\left(\nabla^{\mu} \nabla^{\nu} \pi^{b}\right)
$$


and thus the higher order time derivative terms $\ddot{\pi}^{a} \ddot{\pi}^{b}$ are cancelled out to render our theory stable against ghost instabilities arising from higher order derivatives.

\section{Primordial Perturbation Spectra}

\subsection{Linear Perturbation}

At the level of lowest dimensional operators, the 3 Goldstone bosons are non-dynamical and can be integrated out. After that, the only dynamical degrees are two tensor modes and one scalar mode from inflaton. We use ADM formalism to decompose metric,

$$
d s^{2}=-N^{2} d t^{2}+h_{i j}\left(N^{i} d t+d x^{i}\right)\left(N^{j} d t+d x^{j}\right)
$$

where, neglecting vector perturbations since they are non-dynamical as we have pointed out in the previous section, one has

$$
N=1+\alpha, \quad N_{i}=\partial_{i} \beta, \quad h_{i j}=a^{2} e^{2 \mathcal{R}} \exp \left(\gamma_{i j}+\partial_{i} \partial_{j} E\right)
$$

where $\alpha, \beta, \mathcal{R}, E$ are scalar metric perturbation, and $\gamma_{i j}$ is the tensor perturbation which satisfies transverse and traceless condition $\gamma_{i}^{i}=\partial_{i} \gamma^{i}{ }_{j}=0$. On the other hand, we decompose the scalar fields as

$$
\phi=\phi_{0}+\delta \phi \quad \text { and } \quad \varphi^{i}=x^{i}+\partial^{i} \pi,
$$

where again we only focused on the scalar component. From now on we work in the uniform $\phi$ slicing, i.e. we set $\delta \phi=0$, and therefore $\mathcal{R}$ corresponds to the comoving curvature perturbation. It should be noted that due to the background $S O(3)$ symmetry, scalar modes and tensor modes completely decouple at linear perturbation level.

Scalar-type perturbation: After solving the constraints for $\alpha$ and $\beta$ at linear perturbation level, which yields

$$
\alpha=\frac{\dot{\mathcal{R}}}{H}, \quad \beta=-\frac{\mathcal{R}}{H}-\frac{a^{2} \epsilon \dot{\mathcal{R}}}{k^{2}}+\frac{1}{2} a^{2} \dot{E}
$$

the scalar action reads

$$
S_{(2)}=M_{p}^{2} \int d t d^{3} x a^{3} \epsilon\left(\dot{\mathcal{R}}^{2}-a^{-2}\left(\partial_{i} \mathcal{R}\right)^{2}\right)-\frac{1}{12} m_{g}^{2} k^{4} a^{3}(E-\pi)^{2},
$$

where $\epsilon=-\dot{H} / H^{2}$ is the slow-roll parameter. The graviton mass term gives us an addition constraint. For $\pi=0$, i.e. in the so-called unitary gauge, we obtain $E=0$ by solving the constraint ${ }^{2}$. After integrating out the $E$ component, Eq. (3.5) can be rewritten in terms of the conformal time,

$$
S_{(2)}=\frac{1}{2} \int d \tau d^{3} x z^{2}\left(\mathcal{R}^{\prime 2}-\left(\partial_{i} \mathcal{R}\right)^{2}\right) ; \quad z^{2} \equiv 2 M_{p}^{2} a^{2} \epsilon,
$$

\footnotetext{
${ }^{2}$ With higher order derivative term such as $\nabla^{\mu} \bar{\delta} Z^{i j} \partial_{\mu} \bar{\delta} Z^{i j}$ included, the mode $E$ becomes dynamical again but with mass $m_{E}^{2} \sim M_{p} m_{g} \gg H^{2}$. It decays exponentially on the inflationary background and thus we can neglect its contribution to the perturbation spectra. This is also the case for the vector perturbations.
} 
where the prime' denotes the derivative with respect to the conformal time $d \tau=d t / a(t)$.

In canonical quantization, we write

$$
\mathcal{R}=\int \frac{d^{3} k}{(2 \pi)^{3 / 2}}\left(a_{\boldsymbol{k}} u_{k}(\tau) e^{i \boldsymbol{k} \cdot \boldsymbol{x}}+\text { h.c. }\right),
$$

where $u_{k}(\tau)$ is the positive frequency mode function which satisfies the Klein-Gordon normalization,

$$
u_{k} u_{k}^{* \prime}-u_{k}^{*} u_{k}^{\prime}=\frac{i}{z^{2}}
$$

The equation of motion reads

$$
u_{k}^{\prime \prime}+\frac{\left(z^{2}\right)^{\prime}}{z^{2}} u_{k}^{\prime}+k^{2} u_{k}=0
$$

In the de-Sitter approximation where $a=-1 / H \tau$ and $\epsilon=$ const., which is valid for $k \gg H a$, we have $\left(z^{2}\right)^{\prime} / z^{2}=-2 / \tau$. In the high frequency limit the positive frequency function is given by

$$
u_{k} \rightarrow \frac{1}{z \sqrt{2 k}} e^{-i k \tau}
$$

Then we obtain the solution in the de-Sitter approximation as

$$
u_{k}=\frac{H}{M_{p} \sqrt{4 \epsilon k^{3}}} e^{-i k \tau}(1+i k \tau)
$$

where we have ignored an irrelevant overall phase. For $k \ll H a$, the de Sitter approximation breaks down, but it is easy to see that $u_{k}$ approaches a constant,

$$
u_{k} \rightarrow \frac{H_{k}}{M_{p} \sqrt{4 \epsilon_{k} k^{3}}} e^{-i k \theta_{k}},
$$

where $H_{k}$ and $\epsilon_{k}$ are those evaluated at horizon crossing $-k \tau=1$ and $\theta_{k}$ is a constant phase. We define the Fourier space $\mathcal{R}$ by

$$
\mathcal{R}(\boldsymbol{k}, \tau)=\int d^{3} x \mathcal{R}(\boldsymbol{x}, \tau) e^{-i \boldsymbol{k} \cdot \boldsymbol{x}},
$$

hence

$$
\mathcal{R}(\boldsymbol{k}, \tau)=(2 \pi)^{3 / 2}\left(a_{\boldsymbol{k}} u_{k}(\tau)+a_{-\boldsymbol{k}}^{\dagger} u_{k}^{*}(\tau)\right) .
$$

From now on, we work on the Fourier space, and use the same symbol $\mathcal{R}$ for the Fourier mode unless there is a chance of confusion. The two-point function for $\mathcal{R}$ is given by

$$
\left\langle\mathcal{R}\left(\boldsymbol{k}_{1}, \tau_{1}\right) \mathcal{R}\left(\boldsymbol{k}_{2}, \tau_{2}\right)\right\rangle=(2 \pi)^{3} \delta\left(\boldsymbol{k}_{1}+\boldsymbol{k}_{2}\right) G_{k_{1}}\left(\tau_{1}, \tau_{2}\right),
$$

where

$$
G_{k}\left(\tau_{1}, \tau_{2}\right)=u_{k}\left(\tau_{1}\right) u_{k}^{*}\left(\tau_{2}\right)
$$


Tensor-type perturbation: In contrast to General Relativity (GR), the tensor modes receive a mass correction due to the broken spatial symmetry,

$$
S_{T}^{(2)}=\frac{M_{p}^{2}}{8} \int d t d^{3} x a^{3}\left[\dot{\gamma}_{i j} \dot{\gamma}^{i j}-\left(\frac{k^{2}}{a^{2}}+m_{g}^{2}\right) \gamma_{i j} \gamma^{i j}\right]
$$

or in terms of the conformal time,

$$
S_{T}^{(2)}=\frac{M_{p}^{2}}{8} \int d \tau d^{3} x a^{2}\left[\gamma_{i j}^{\prime} \gamma^{i j \prime}-\left(k^{2}+m_{g}^{2} a^{2}\right) \gamma_{i j} \gamma^{i j}\right]
$$

Noted that we have neglected the contributions from higher order derivatives discussed in the previous section since they are small. As before we decompose the field into its Fourier modes,

$$
\gamma_{i j}(x)=\int \frac{d^{3} k}{(2 \pi)^{3}} \sum_{s= \pm} \epsilon_{i j}^{s}(\boldsymbol{k}) \gamma^{s}(\boldsymbol{k}, \tau) e^{i \boldsymbol{k} \cdot \boldsymbol{x}} ; \quad \gamma^{s}(\boldsymbol{k}, \tau)=(2 \pi)^{3 / 2}\left[b_{\boldsymbol{k}}^{s} \gamma_{k}+b_{-\boldsymbol{k}}^{s} \gamma_{k}^{*}\right]
$$

where $b_{\boldsymbol{k}}^{s}$ is the annihilation operator and $s$ is the polarisation index. The polarization tensor $\epsilon_{i j}^{s}$ obeys

$$
\epsilon_{i i}^{s}(\boldsymbol{k})=k^{i} \epsilon_{i j}^{s}(\boldsymbol{k})=0, \quad \epsilon_{i j}^{s *}(\boldsymbol{k})=\epsilon_{i j}^{-s}(\boldsymbol{k})=\epsilon_{i j}^{s}(-\boldsymbol{k}), \quad \epsilon_{i j}^{s}(\boldsymbol{k}) \epsilon_{i j}^{s^{\prime}}(\boldsymbol{k})=2 \delta_{s,-s^{\prime}} .
$$

The action (3.17) yields the equation of motion

$$
\gamma_{k}^{\prime \prime}+\frac{\left(a^{2}\right)^{\prime}}{a^{2}} \gamma_{k}^{\prime}+\left(k^{2}+m_{g}^{2} a^{2}\right) \gamma_{k}=0
$$

where again the positive frequency mode function satisfies the Klein-Gordon normalizaion,

$$
\gamma_{k} \gamma_{k}^{* \prime}-\gamma_{k}^{*} \gamma_{k}^{\prime}=\frac{i}{2 a^{2}}
$$

where the factor 2 in the denominator is due to the factor 2 in the normalization of the polarization tensor defined in Eq. (3.20).

Below we assume $m_{g}^{2} \ll H^{2}$ (although interesting physics may arise when $m_{g}^{2} \gtrsim H^{2}$, similar to the case studied in [48-51]). Assuming the Bunch-Davies vacuum initial condition, the solution to Eq. (3.21) under the de Sitter approximation is given by a Hankel function of the first kind,

$$
\gamma_{k}=\frac{H}{M_{p}} \sqrt{\frac{\pi}{2 k^{3}}}(-k \tau)^{3 / 2} H_{\nu_{g}}^{(1)}(-k \tau)
$$

where $\nu_{g}=\sqrt{9 / 4-m_{g}^{2} / H^{2}}$. In the massless limit $m_{g}^{2} \rightarrow 0$, the solution reduces to

$$
\gamma_{k}=\frac{i H}{M_{p} \sqrt{k^{3}}} e^{-i k \tau}(1+i k \tau)
$$

In the case of a small but non-vanishing graviton mass, $m_{g}^{2} / H^{2} \ll 1$, we find on superhorizon scales

$$
\gamma_{k} \simeq \frac{H_{k}}{M_{p} \sqrt{k^{3}}}(-k \tau)^{m_{g}^{2} / 3 H^{2}} .
$$


Note that, as opposed to GR, the tensor modes are no longer constant on superhorizon scales. It gains a tiny time dependence. The larger the graviton mass is, the faster it decays. One can easily see this behavior by solving Eq. (3.21) in the limit $k^{2} \rightarrow 0$ in terms of the number of e-folds, that is $d N=a H d \tau$. The power spectrum of primordial gravitational waves is thus calculated as

$$
P_{\gamma}=\frac{2 H^{2}}{\pi^{2} M_{p}^{2}}\left(\frac{k}{a H}\right)^{n_{t}},
$$

with a tilt

$$
n_{t} \simeq-2 \epsilon+\frac{2 m_{g}^{2}}{3 H^{2}}
$$

Interestingly if $m_{g}^{2}>3 H^{2} \epsilon$, the primordial gravitational waves have a blue tilt, which could be a smoking gun of massive gravity in the early universe (for other possibilities of blue tilted tensor modes, see [52]). Similar to the scalar mode, it is useful to define the tensor two-point function

$$
\left\langle\gamma^{s}\left(\boldsymbol{k}_{1}, \tau_{1}\right) \gamma^{\tilde{s}}\left(\boldsymbol{k}_{2}, \tau_{2}\right)\right\rangle=(2 \pi)^{3} \delta\left(\boldsymbol{k}_{1}+\boldsymbol{k}_{2}\right) W_{k_{1}}\left(\tau_{1}, \tau_{2}\right) \delta_{s \tilde{s}}
$$

where

$$
W_{k}\left(\tau_{1}, \tau_{2}\right)=\gamma_{k}\left(\tau_{1}\right) \gamma_{k}^{*}\left(\tau_{s}\right)
$$

It should be noted that, under the small mass approximation $m_{g}^{2} \ll H^{2}$, as in the single field case, the tensor to scalar ratio is given by

$$
r \equiv \lim _{-k \tau \ll 1} \frac{2 \times 2 W_{k}(\tau, \tau)}{G_{k}(\tau, \tau)} \sim 16 \epsilon,
$$

where the additional factor 2 on the right-hand side is again due to our normalization of the polarization tensor (3.20).

\subsection{Non-linear Perturbations}

At cubic action level, the graviton mass term in the action eq. (2.5) reads

$$
\begin{aligned}
S_{g} & =-\frac{9}{8} \int d^{4} x \sqrt{-g} M_{p}^{2} m_{g}^{2} \bar{\delta} Z^{i j} \bar{\delta} Z^{i j} \\
& \supset M_{p}^{2} m_{g}^{2} \int-\frac{1}{4} a^{5} \gamma_{i j} N^{i} N^{j}-\frac{1}{8} a^{3}(\alpha+3 \mathcal{R}) \gamma_{i j} \gamma_{i j}+\frac{3}{8} a^{3} \gamma_{i j} \gamma_{j k} \gamma_{k i} .
\end{aligned}
$$

There are three types of interactions, the first term of the above equation is scalar-scalar-tensor, the second term is scalar-tensor-tensor, and the last term is with 3 tensors. In our current work, we focus on the CMB temperature and E-mode polarisation non-Gaussianities arising from primordial gravitational waves, provided that primordial three-scalar interaction is negligible. Noted that after inflation, gravitational waves decay as $a^{-1}$ after re-entering the cosmic horizon, while scalar perturbation underwent baryon acoustic oscillations, and co-evolve with matter sector at late time. Naively we would expect that the contributions from the above three types primordial coupling scales as $a^{-1}, a^{-2}$ and $a^{-3}$ respectively. Therefore, on the observational scales that we are interested, we consider the primordial scalar-scalartensor coupling as the dominant contribution to the CMB temperature and E-mode polarisation nonGaussianities. 
Plug in the solution of the momentum constraint Eq. (3.4), the leading contribution in the scalarscalar-tensor interaction is given by

$$
S_{s s t}=-\frac{1}{4} M_{p}^{2} \int a^{3} \frac{m_{g}^{2}}{H^{2}} \gamma_{i j} \partial_{i} \mathcal{R} \partial_{j} \mathcal{R}+\ldots
$$

We have learned that the non-trivial scalar-scalar-tensor correlation arises from the coupling term such as $\gamma_{i j} N^{i} N^{j}$ in Eq. (3.31). In fact, such a term also arises from the cubic coupling between the spacelike Stueckelberg fields and the inflaton scalar field,

$$
-\frac{1}{2} \lambda \int \sqrt{-g} \frac{\bar{\delta} Z^{i j}}{Z} \cdot \partial^{\mu} \varphi^{i} \partial_{\mu} \phi \cdot \partial^{\nu} \varphi^{j} \partial_{\nu} \phi=-\lambda M_{p}^{2} H^{2} \epsilon \int a^{5} \gamma_{i j} N^{i} N^{j},
$$

where $\lambda$ is a free parameter of our low energy effective field theory, whose origin can be understood only after we have UV completion of our theory. Here we do not discuss this issue since it is beyond the scope of the current work. Including the contribution from graviton mass term, Eq. (3.31), the scalar-scalar-tensor coupling term takes the form,

$$
S^{(3)} \supset-\lambda_{s s t} M_{p}^{2} H^{2} \epsilon \int a^{3}\left(\frac{1}{a^{2} H^{2}} \gamma_{i j} \partial_{i} \mathcal{R} \partial_{j} \mathcal{R}-\frac{2 \epsilon}{H} \gamma_{i j} \partial_{i} \mathcal{R} \partial_{j} \partial^{-2} \dot{\mathcal{R}}+a^{2} \epsilon^{2} \gamma_{i j} \partial_{i} \partial^{-2} \dot{\mathcal{R}} \partial_{j} \partial^{-2} \dot{\mathcal{R}}\right),
$$

where

$$
\lambda_{s s t}=\frac{m_{g}^{2}}{4 H^{2} \epsilon}+\lambda .
$$

\subsection{Primordial 3-point Correlation Function}

The interaction Hamiltonian reads

$$
H_{i n t}=-L_{i n t}=\lambda_{s s t} M_{p}^{2} H^{2} \epsilon a^{3}\left(\frac{1}{a^{2} H^{2}} \gamma_{i j} \partial_{i} \mathcal{R} \partial_{j} \mathcal{R}-\frac{2 \epsilon}{H} \gamma_{i j} \partial_{i} \mathcal{R} \partial_{j} \partial^{-2} \dot{\mathcal{R}}+a^{2} \epsilon^{2} \gamma_{i j} \partial_{i} \partial^{-2} \dot{\mathcal{R}} \partial_{j} \partial^{-2} \dot{\mathcal{R}}\right)
$$

and the 3-point function can thus be calculated by means of the in-in formalism as

$$
\begin{aligned}
& \left\langle\gamma_{\boldsymbol{k}_{1}}^{s} \mathcal{R}_{\boldsymbol{k}_{2}} \mathcal{R}_{\boldsymbol{k}_{3}}\right\rangle_{1} \\
= & 2 ! \times \operatorname{Re}\left[2 i \lambda_{s s t} M_{p}^{2} H^{2} \epsilon \int_{-\infty(1-i \epsilon)}^{\tau} \frac{d \tilde{\tau}}{\tilde{\tau}^{2}}\left\langle 0\left|\gamma_{\boldsymbol{k}_{1}}^{s}(\tau) \mathcal{R}_{\boldsymbol{k}_{2}}(\tau) \mathcal{R}_{\boldsymbol{k}_{3}}(\tau) \gamma_{\boldsymbol{k}_{4}}^{s}(\tilde{\tau}) \epsilon_{i j}^{s} k_{5 i} k_{6 j} \mathcal{R}_{\boldsymbol{k}_{5}}(\tilde{\tau}) \mathcal{R}_{\boldsymbol{k}_{6}}(\tilde{\tau})\right| 0\right\rangle\right]_{\tau \rightarrow 0} \\
= & 2 ! \times \operatorname{Re}\left[2 i \lambda_{s s t} M_{p}^{2} H^{2} \epsilon \epsilon_{i j}^{-s} k_{2 i} k_{3 j} \int_{-\infty(1-i \epsilon)}^{0} \frac{d \tilde{\tau}}{\tilde{\tau}^{2}} W_{k_{1}}(0, \tilde{\tau}) G_{k_{2}}(0, \tilde{\tau}) G_{k_{3}}(0, \tilde{\tau})\right] \\
= & -(2 \pi)^{3} \delta^{(3)}\left(\sum \boldsymbol{k}_{i}\right) \cdot \frac{1}{\Pi\left(k_{i}\right)^{3}} \cdot \frac{\lambda_{s s t} H^{4}}{4 \epsilon M_{p}^{4}} \cdot\left(-k_{t}+\frac{\sum_{i<j} k_{i} k_{j}}{k_{t}}+\frac{k_{1} k_{2} k_{3}}{k_{t}^{2}}\right) \epsilon_{i j}^{-s} k_{2 i} k_{3 j} .
\end{aligned}
$$

where $k_{t} \equiv k_{1}+k_{2}+k_{3}$, and the subscript "1" of $\langle\ldots\rangle_{1}$ stands for the 1 st term in the parentheses on the right-hand side of Eq. (3.36). We have taken the massless approximation $m_{g}^{2} / H^{2} \ll 1$ to compute the above integral. Noted that this term is the same as the one from the cubic scalar interaction in single-field slow-roll inflation in the context of Einstein gravity[53], except for the overall coefficient $\lambda_{\text {sst }}$. A similar term has also been spotted in an extended effective field theory of inflation [54]. It may dominate over 
the cubic scalar contribution and thus leave an imprint on the CMB non-Gaussianity for a sufficiently large $\lambda_{\text {sst }}$.

The contribution from the next-to-leading order in slow-roll parameter (2nd term in the parentheses) reads

$$
\begin{aligned}
& \left\langle\gamma_{\boldsymbol{k}_{1}}^{s} \mathcal{R}_{\boldsymbol{k}_{2}} \mathcal{R}_{\boldsymbol{k}_{3}}\right\rangle_{2} \\
= & \operatorname{Re}\left[2 i \lambda_{s s t} M_{p}^{2} H^{2} \epsilon \int_{-\infty(1-i \epsilon)}^{\tau} \frac{2 \epsilon}{H(-H \tilde{\tau})^{3}} d \tilde{\tau}\left\langle 0\left|\gamma_{\boldsymbol{k}_{1}}^{s}(\tau) \mathcal{R}_{\boldsymbol{k}_{2}}(\tau) \mathcal{R}_{\boldsymbol{k}_{3}}(\tau) \gamma_{\boldsymbol{k}_{4}}^{s}(\tilde{\tau}) \epsilon_{i j}^{s} k_{5 i} k_{6 j} k_{6}^{-2} \mathcal{R}_{\boldsymbol{k}_{5}}(\tilde{\tau}) \mathcal{R}_{\boldsymbol{k}_{6}}^{\prime}(\tilde{\tau})\right| 0\right\rangle\right]_{\tau \rightarrow 0} \\
= & (2 \pi)^{3} \delta^{(3)}\left(\sum \boldsymbol{k}_{i}\right) \cdot \frac{1}{4} \lambda_{s s t} \frac{H^{4}}{M_{p}^{4}} \log \left(-k_{t} \tau\right) \epsilon_{i j}^{-s} k_{2 i} k_{3 j}\left(\frac{1}{k_{1}^{3} k_{2}^{3} k_{3}^{2}}+\frac{1}{k_{1}^{3} k_{2}^{2} k_{3}^{3}}\right),
\end{aligned}
$$

where $\log \left(-k_{t} \tau\right) \simeq N_{k_{t}}$ is the number of e-foldings from the time of horizon crossing to the end of inflation. Typically $N_{k_{t}} \simeq 50 \sim 60$ for observable cosmological large scales. The last term in the parentheses is suppressed by $\epsilon^{2}$ and thus irrelevant.

Summing up Eqs. (3.37) and (3.38) we get

$$
\begin{aligned}
& \left\langle\gamma_{\boldsymbol{k}_{1}}^{s} \mathcal{R}_{\boldsymbol{k}_{2}} \mathcal{R}_{\boldsymbol{k}_{3}}\right\rangle \\
\simeq & (2 \pi)^{3} \delta^{(3)}\left(\sum \boldsymbol{k}_{i}\right) \cdot \lambda_{s s t} \frac{H^{4}}{M_{p}^{4}} \cdot \frac{\epsilon_{i j}^{-s} k_{2 i} k_{3 j}}{\Pi\left(k_{i}\right)^{3}} \cdot\left[-\frac{1}{4 \epsilon}\left(-k_{t}+\frac{\sum_{i<j} k_{i} k_{j}}{k_{t}}+\frac{k_{1} k_{2} k_{3}}{k_{t}^{2}}\right)+\frac{N_{k_{t}}}{4}\left(k_{2}+k_{3}\right)\right] .
\end{aligned}
$$

This contribution should be compared to that coming from the 3 -scalar interaction in GR. Recall that such terms are given by $[53,55]$

$$
S_{3 s}=M_{p}^{2} \int \epsilon^{2} a^{3} \mathcal{R} \dot{\mathcal{R}}^{2}+\ldots \quad \text { and } \quad\left\langle\mathcal{R}_{\boldsymbol{k}_{1}} \mathcal{R}_{\boldsymbol{k}_{2}} \mathcal{R}_{\boldsymbol{k}_{3}}\right\rangle \sim \frac{H^{4}}{\epsilon}
$$

Then the ratio between $\left\langle\gamma_{\boldsymbol{k}_{1}}^{s} \mathcal{R}_{\boldsymbol{k}_{2}} \mathcal{R}_{\boldsymbol{k}_{3}}\right\rangle$ and $\left\langle\mathcal{R}_{\boldsymbol{k}_{1}} \mathcal{R}_{\boldsymbol{k}_{2}} \mathcal{R}_{\boldsymbol{k}_{3}}\right\rangle$ is given by

$$
\frac{\left\langle\gamma_{\boldsymbol{k}_{1}}^{s} \mathcal{R}_{\boldsymbol{k}_{2}} \mathcal{R}_{\boldsymbol{k}_{3}}\right\rangle}{\left\langle\mathcal{R}_{\boldsymbol{k}_{1}} \mathcal{R}_{\boldsymbol{k}_{2}} \mathcal{R}_{\boldsymbol{k}_{3}}\right\rangle} \sim \lambda_{s s t}
$$

where we used Eq. (3.39). Thus, the scalar-scalar-tensor contribution dominates over the scalar-scalarscalar contribution if $\lambda_{s s t}>1$. Noting that the non-Gaussianity arising from $\left\langle\mathcal{R}_{\boldsymbol{k}_{1}} \mathcal{R}_{\boldsymbol{k}_{2}} \mathcal{R}_{\boldsymbol{k}_{3}}\right\rangle$ is of the order of the slow-roll parameter $\epsilon$, i.e. $f_{N L}^{s s s} \sim \epsilon$, we can estimate the non-Gaussianity from $\left\langle\gamma_{\boldsymbol{k}_{1}}^{s} \mathcal{R}_{\boldsymbol{k}_{2}} \mathcal{R}_{\boldsymbol{k}_{3}}\right\rangle$ as

$$
f_{N L}^{s s t} \sim \lambda_{s s t} \cdot \epsilon
$$

Thus $\lambda_{\text {sst }} \gtrsim \mathcal{O}\left(10^{3}\right)$ will be required for the temperature and polarization bispectra to be observed if $\epsilon \sim 10^{-3}$. This will be quantitatively estimated in the next section where we perform numerical computations. 


\section{CMB Bispectra}

We now analyze the signatures of the primordial scalar-scalar-tensor correlator (3.39) in the CMB temperature and polarization bispectra. For convenience, we rewrite Eq. (3.39) as

$$
\begin{aligned}
\left\langle\gamma_{\mathbf{k}_{1}}^{\lambda} \mathcal{R}_{\mathbf{k}_{2}} \mathcal{R}_{\mathbf{k}_{3}}\right\rangle & =(2 \pi)^{3} \delta^{(3)}\left(\mathbf{k}_{1}+\mathbf{k}_{2}+\mathbf{k}_{3}\right) \epsilon_{i j}^{-\lambda}\left(\mathbf{k}_{1}\right) \hat{k}_{2 i} \hat{k}_{3 j} F_{k_{1} k_{2} k_{3}}^{(t s s)}, \\
F_{k_{1} k_{2} k_{3}}^{(t s s)} & \equiv-\frac{16 \pi^{4} \lambda_{s s t} \epsilon A_{S}^{2}}{k_{1}^{2} k_{2}^{2} k_{3}^{2}}\left[\frac{I_{k_{1} k_{2} k_{3}}}{k_{t}} \frac{k_{t}}{k_{1}}-\epsilon N_{\text {tot }} \frac{k_{2}+k_{3}}{k_{1}}\right], \\
I_{k_{1} k_{2} k_{3}} & \equiv-k_{t}+\frac{k_{1} k_{2}+k_{2} k_{3}+k_{3} k_{1}}{k_{t}}+\frac{k_{1} k_{2} k_{3}}{k_{t}^{2}}
\end{aligned}
$$

where $A_{S} \equiv H^{2} /\left(8 \pi^{2} \epsilon M_{p}^{2}\right)$, and we have assumed that $N_{k_{t}}=N_{\text {tot }}=$ const for simplicity.

At linear order, the harmonic coefficients of the CMB anisotropies, $a_{\ell m}=\int d^{2} \hat{\mathbf{n}} X(\hat{\mathbf{n}}) Y_{\ell m}^{*}(\hat{\mathbf{n}})$, from $\mathcal{R}$ and $\gamma^{ \pm}$are generally expressed according to $[56,57]$ as

$$
\begin{aligned}
& a_{\ell m}^{(s) X}=4 \pi i^{\ell} \int \frac{d^{3} k}{(2 \pi)^{3}} \mathcal{T}_{\ell(s)}^{X}(k) \mathcal{R}_{\mathbf{k}} Y_{\ell m}^{*}(\hat{\mathbf{k}}), \\
& a_{\ell m}^{(t) X}=4 \pi i^{\ell} \int \frac{d^{3} k}{(2 \pi)^{3}} \mathcal{T}_{\ell(t)}^{X}(k) \sum_{\lambda= \pm} \lambda^{x} \gamma_{\mathbf{k}-2 \lambda}^{\lambda} Y_{\ell m}^{*}(\hat{\mathbf{k}}),
\end{aligned}
$$

where $\mathcal{T}_{\ell(z)}^{X}$ denotes the scalar-mode $(z=s)$ and tensor-mode $(z=t)$ radiation transfer functions for the temperature $(X=T)$ and the E/B-mode polarization $(X=E / B)$, and $x=0$ for $X=T, E$ and $x=1$ for $X=B$. Note that $a_{\ell m}^{(s) B}=0$. From these equations, we can formulate the induced CMB scalar-scalar-tensor bispectra as

$$
\begin{aligned}
\left\langle a_{\ell_{1} m_{1}}^{(t) X_{1}} a_{\ell_{2} m_{2}}^{(s) X_{2}} a_{\ell_{3} m_{3}}^{(s) X_{3}}\right\rangle= & {\left[\prod_{n=1}^{3} 4 \pi i^{\ell_{n}} \int_{0}^{\infty} \frac{k_{n}^{2} d k_{n}}{(2 \pi)^{3}}\right] \mathcal{T}_{\ell_{1}(t)}^{X_{1}}\left(k_{1}\right) \mathcal{T}_{\ell_{2}(s)}^{X_{2}}\left(k_{2}\right) \mathcal{T}_{\ell_{3}(s)}^{X_{3}}\left(k_{3}\right) F_{k_{1} k_{2} k_{3}}^{(t s s)} } \\
& {\left[\prod_{n=1}^{3} \int d^{2} \hat{\mathbf{k}}_{n}\right] \sum_{\lambda_{1}= \pm} \lambda_{1}^{x_{1}}-_{2 \lambda_{1}} Y_{\ell_{1} m_{1}}^{*}\left(\hat{\mathbf{k}}_{1}\right) Y_{\ell_{2} m_{2}}^{*}\left(\hat{\mathbf{k}}_{2}\right) Y_{\ell_{3} m_{3}}^{*}\left(\hat{\mathbf{k}}_{3}\right) } \\
& (2 \pi)^{3} \delta^{(3)}\left(\mathbf{k}_{1}+\mathbf{k}_{2}+\mathbf{k}_{3}\right) \epsilon_{i j}^{-\lambda_{1}}\left(\mathbf{k}_{1}\right) \hat{k}_{2 i} \hat{k}_{3 j} .
\end{aligned}
$$

Employing the harmonic-space expressions of $\delta^{(3)}\left(\mathbf{k}_{1}+\mathbf{k}_{2}+\mathbf{k}_{3}\right) \epsilon_{i j}^{-\lambda_{1}}\left(\mathbf{k}_{1}\right) \hat{k}_{2 i} \hat{k}_{3 j}$ and the law of addition of angular momentum, the $\hat{\mathbf{k}}_{1,2,3}$ integrals in the 2 nd and 3rd lines are analytically performed and expressed in terms of the products of the Wigner $3 j$ and $9 j$ symbols. Since the same angular integrals are realized in the model studied in Ref.[56] (mathematically equivalent to the case for $N_{\text {tot }}=0$ ), we make use of their results, which leads us to

$$
\begin{aligned}
& \left\langle a_{\ell_{1} m_{1}}^{(t) X_{1}} a_{\ell_{2} m_{2}}^{(s) X_{2}} a_{\ell_{3} m_{3}}^{(s) X_{3}}\right\rangle=B_{\ell_{1} \ell_{2} \ell_{3}}^{(t s s) X_{1} X_{2} X_{3}}\left(\begin{array}{ccc}
\ell_{1} & \ell_{2} & \ell_{3} \\
m_{1} & m_{2} & m_{3}
\end{array}\right),
\end{aligned}
$$

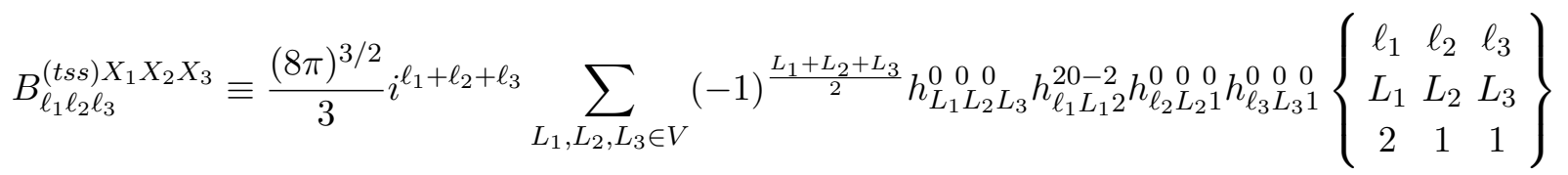

$$
\begin{aligned}
& \times \int_{0}^{\infty} y^{2} d y \frac{2}{\pi} \int_{0}^{\infty} k_{1}^{2} d k_{1} \mathcal{T}_{\ell_{1}(t)}^{X_{1}} j_{L_{1}}\left(k_{1} y\right)\left[\prod_{n=2}^{3} \frac{2}{\pi} \int_{0}^{\infty} k_{n}^{2} d k_{n} \mathcal{T}_{\ell_{n}(s)}^{X_{n}} j_{L_{n}}\left(k_{n} y\right)\right] F_{k_{1} k_{2} k_{3}}^{(t s s)},
\end{aligned}
$$



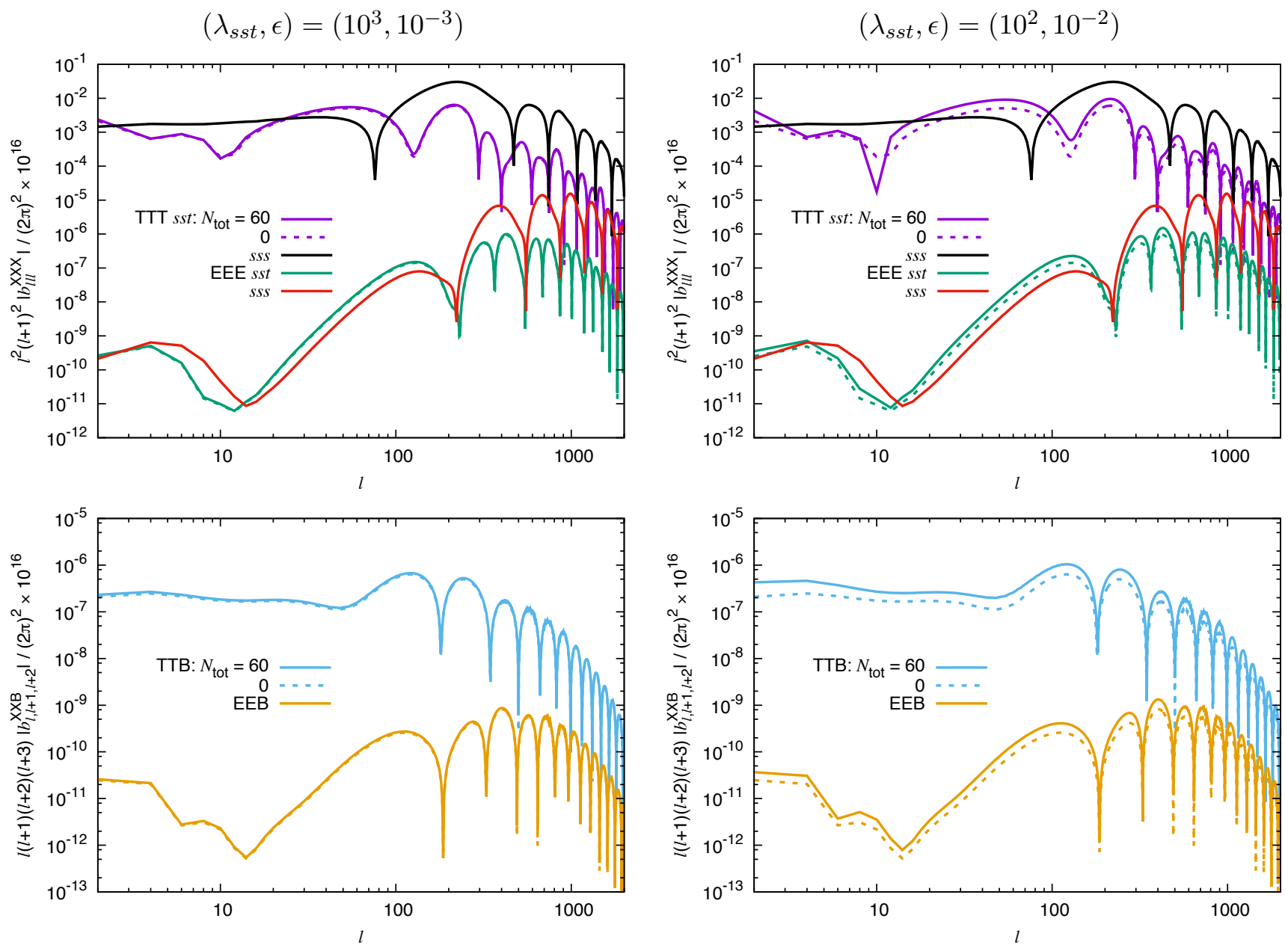

Figure 1. Equilateral-limit shapes of the TTT and EEE bispectra (top two panels), given by $b_{\ell_{1} \ell_{2} \ell_{3}}^{X X X}=$ $\left[B_{\ell_{1} \ell_{2} \ell_{3}}^{(t s) X X X}+B_{\ell_{1} \ell_{2} \ell_{3}}^{(s t s X X}+B_{\ell_{1} \ell_{2} \ell_{3}}^{(s s t) X X}\right] / h_{\ell_{1} \ell_{2} \ell_{3}}^{00}$, and the TTB and EEB bispectra (bottom two panels), given by $b_{\ell_{1} \ell_{2} \ell_{3}}^{X X X}=B_{\ell_{1} \ell_{2} \ell_{3} X B}^{(s s t) X h_{\ell_{1}+2, \ell_{2}+1, \ell_{3}}^{0}}$, from the sst correlator $(3.39)$ with $\left(\lambda_{s s t}, \epsilon\right)=\left(10^{3}, 10^{-3}\right)$ and $\left(10^{2}, 10^{-2}\right)$. Solid (dotted) line corresponds to the case for $N_{\text {tot }}=60$ (0). For comparison, in the top panels we also plot the TTT and EEE bispectra in the sss case with $f_{\mathrm{NL}}^{s s s}=1$.

where $h_{l_{1} l_{2} l_{3}}^{s_{1} s_{2} s_{3}} \equiv \sqrt{\frac{\left(2 l_{1}+1\right)\left(2 l_{2}+1\right)\left(2 l_{3}+1\right)}{4 \pi}}\left(\begin{array}{lll}l_{1} & l_{2} & l_{3} \\ s_{1} & s_{2} & s_{3}\end{array}\right)$, and the $\ell$-space domain $V$ corresponds to

$$
L_{1}=\left\{\begin{array}{ll}
\left|\ell_{1} \pm 2\right|, \ell_{1} & \left(\text { for } X_{1}=T, E\right) \\
\left|\ell_{1} \pm 1\right| & \left(\text { for } X_{1}=B\right)
\end{array} \quad, \quad L_{2}=\left|\ell_{2} \pm 1\right|, \quad L_{3}=\left|\ell_{3} \pm 1\right|\right.
$$

Using this formula, in what follows, we numerically analyze two parity-even correlators: TTT and EEE, and two parity-odd ones: TTB and EEB with $A_{S}=2.4 \times 10^{-9}$. We then employ an approximation, $\frac{I_{k_{1} k_{2} k_{3}}}{k_{t}} \approx-0.65$, to find a factorized form $F_{k_{1} k_{2} k_{3}}^{(t s s)} \approx \sum_{a b c} A_{a}\left(k_{1}\right) B_{b}\left(k_{2}\right) C_{c}\left(k_{3}\right)$ to reduce the computational cost.

Figure 1 shows the equilateral-limit shapes of the TTT, EEE, TTB and EEB bispectra from the scalarscalar-tensor correlator (referred to as sst) for several $\left(\lambda_{s s t}, \epsilon, N_{\text {tot }}\right)$. Here the TTT and EEE (TTB and 

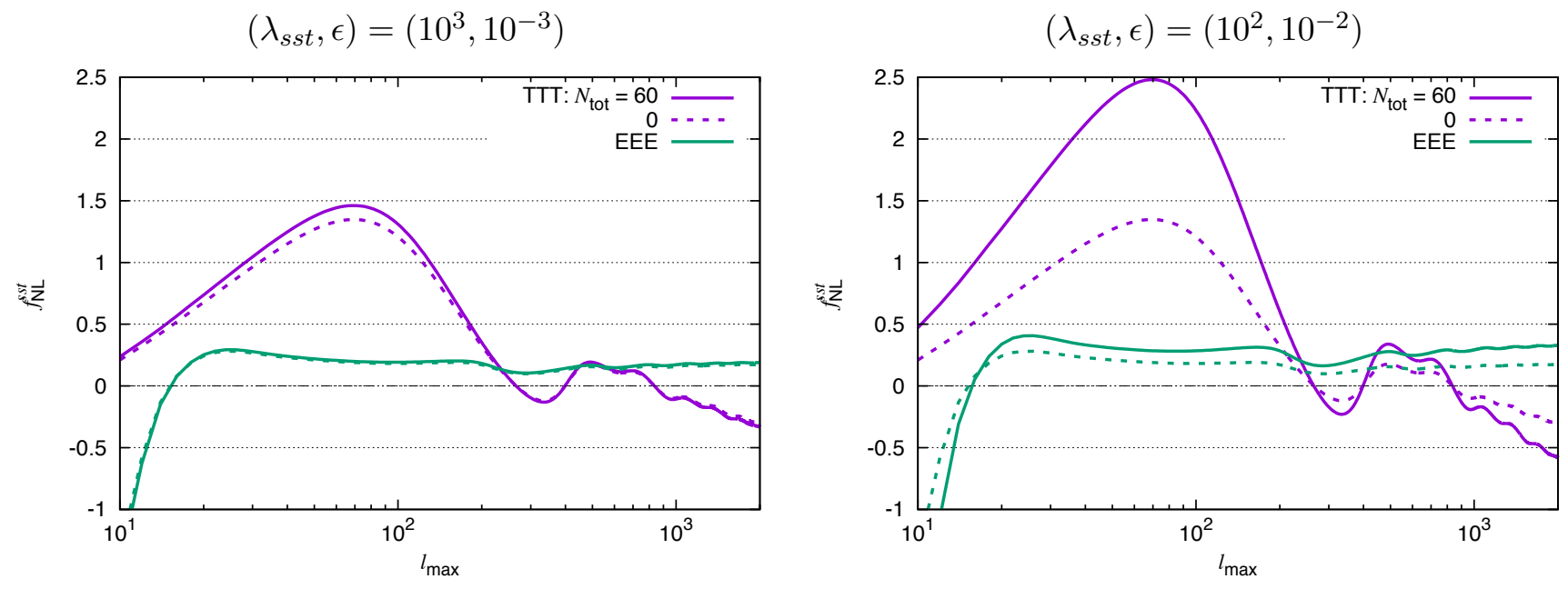

Figure 2. Size of $f_{\mathrm{NL}}^{s s s}$ translated from the sst correlator, i.e., $f_{\mathrm{NL}}^{s s t}$, for $\left(\lambda_{s s t}, \epsilon\right)=\left(10^{3}, 10^{-3}\right)$ and $\left(10^{2}, 10^{-2}\right)$, estimated from TTT and EEE. Solid and dotted lines correspond to the cases for $N_{\text {tot }}=60$ and 0 , respectively. Given the value of $\epsilon$ fixed, the vertical axis could be understood as $f_{N L}^{s s t} /\left(\epsilon \lambda_{s s t}\right)$ and thus $f_{N L}^{s s t}$ linearly depends on $\lambda_{s s t}$.

EEB) bispectra obey $\ell_{1}+\ell_{2}+\ell_{3}=$ even (odd) because of no parity violation in the sst correlator (3.39). One can find the expected signatures such as the amplification at $\ell \sim 200$ due to the acoustic oscillation in TTT and TTB, and the reionization bump for $\ell \lesssim 10$ in EEE and EEB. In the top panel, we also draw the TTT and EEE bispectra induced by the usual scalar-mode local-type non-Gaussianity (referred to as sss) with $f_{\mathrm{NL}}^{s s s}=1$ [58]. It can be easily seen that the $s s t$ bispectra are comparable in size to the $s s s$ ones up to $\ell \sim 100$, while, after that, the difference appears due to the rapid decay of the tensor-mode transfer function compared with the scalar-mode one. The overall amplitudes of the bispectra change depending on $\lambda_{s s t}, \epsilon$ and $N_{\text {tot }}$. We cannot, however, see the discrepancy between the bispectra with $N_{\text {tot }}=60$ and 0 when $\epsilon=10^{-3}$, which implies that the contribution of the 2nd term in Eq. (3.39) is subdominant.

To discuss more quantitatively, let us perform the Fisher matrix analysis. Ignoring the contributions of the higher-order correlations to the covariance matrix, the Fisher matrices of XXX and XXB for $\mathrm{X}=\mathrm{T}$ or $E$ are given by

$$
\begin{aligned}
& F_{i j}^{X X X}=\sum_{\ell_{1}, \ell_{2}, \ell_{3}=2}^{\ell_{\max }} \frac{\hat{B}_{\ell_{1} \ell_{2} \ell_{3}}^{X X X, i} \hat{B}_{\ell_{1} \ell_{2} \ell_{3}}^{X X X, j}}{6 C_{\ell_{1}}^{X X} C_{\ell_{2}}^{X X} C_{\ell_{3}}^{X X}}(-1)^{\ell_{1}+\ell_{2}+\ell_{3}}, \\
& F_{i j}^{B X X}=\sum_{\ell_{1}, \ell_{2}, \ell_{3}=2}^{\ell_{\max }} \frac{\hat{B}_{\ell_{1} \ell_{2} \ell_{3}}^{B X X} \hat{B}_{\ell_{1} \ell_{2} \ell_{3}}^{B X, j}}{2 C_{\ell_{1}}^{B B} C_{\ell_{2}}^{X X} C_{\ell_{3}}^{X X}}(-1)^{\ell_{1}+\ell_{2}+\ell_{3}},
\end{aligned}
$$

where $\hat{B}_{\ell_{1} \ell_{2} \ell_{3}}^{i}$ is the bispectrum in the $i$-th model normalized with the amplitude parameter, and $C_{\ell}$ is the power spectrum including experimental uncertainties. We consider noiseless measurements of the temperature and E-mode polarization up to $\ell=2000$ (comparable to the sensitivity in the Planck experiment [33]), so $C_{\ell}^{T T}$ and $C_{\ell}^{E E}$ are determined by the cosmic variance alone. For the TTB and EEB cases, we assume the analyses with the B-mode data (without delensing) expected in a futuristic survey like the LiteBIRD project and thus $C_{\ell}^{B B}$ is given by the sum of the cosmic variance, the lensed B-mode 
spectrum and the LiteBIRD-level noise spectrum $[27,59,60] \cdot{ }^{3}$ Since we are working with the massless approximation $m_{g}^{2} / H^{2} \ll 1$, the cosmic variance is given by the BB power spectrum with the tensor-toscalar ratio $r \simeq 16 \epsilon$. We then consider the full-sky measurements and ignore the contaminations due to residual foregrounds for simplicity.

Using the information of TTT or EEE, we can estimate the size of $f_{\mathrm{NL}}^{s s s}$ translated from the $s s t$ correlator, equivalent to $f_{\mathrm{NL}}^{s s t}$ in Eq. (3.42), according to $f_{\mathrm{NL}}^{s s t}=\lambda_{s s t} F_{s s t, s s s}^{X X X} / F_{s s s, s s s}^{X X X}$. Figure 2 shows the results as a function of $\ell_{\max }$. For the TTT case, the sst bispectrum is weakly correlated to the $s s s$ one for $\ell_{\max } \gtrsim 100$ because, at such scales, the tensor-mode and scalar-mode transfer functions, $\mathcal{T}_{\ell(t)}^{T}(k)$ and $\mathcal{T}_{\ell(s)}^{T}(k)$, have very different shapes [61]. For $\ell_{\max } \lesssim 100$, however, the correlation recovers because of the shape similarity between the sst and sss bispectra due to the (Integrated) Sachs-Wolfe plateau [61]. For this reason, $\left|f_{\mathrm{NL}}^{s s t}\right|$ falls below the theoretical expectation $\sim\left|\epsilon \lambda_{s s t}\right|$ [see Eq. (3.42)] for $\ell_{\max } \gtrsim 100$. For the EEE case, thanks to the weakness of the correlation between the $s s t$ and sss bispectra, $\left|f_{\mathrm{NL}}^{\text {sst }}\right|$ falls below $\left|\epsilon \lambda_{s s t}\right| / 2$ for $\ell_{\max } \gtrsim 20$. As expected from Fig. 1, if $\epsilon=10^{-3}, f_{\mathrm{NL}}^{s s t} / \lambda_{s s t}$ with $N_{\text {tot }}=60$ almost overlaps that with $N_{\text {tot }}=0$.

The top panel of Fig. 3 describes the expected $1 \sigma$ errors on $\lambda_{s s t}$, computed according to $\Delta \lambda_{s s t}=$ $1 / \sqrt{F_{s s t, s s t}}$, in the TTT, EEE, TTB and EEB analyses. From this, we find a $\ell_{\max }$ scaling similar to the sss case; namely $\Delta \lambda_{s s t} \propto \ell_{\max }^{-1}$, in TTT and EEE [56] and a bit more rapid scaling in TTB and EEB. In the case that the 2nd term of Eq. (3.39) contributes weakly to the Fisher matrix, Eq. (4.10) indicates that $\Delta \lambda_{\text {sst }}$ from TTT or EEE simply scales like $\epsilon^{-1}$. Concerning TTB and EEB, in the Fisher matrix (4.11), the dominant contribution comes from the squeezed-limit signal: $\ell_{1} \ll \ell_{2} \sim \ell_{3}$. On such small $\ell_{1}$ 's, $C_{\ell_{1}}^{B B}$ in the denominator is almost determined by the cosmic variance and thus $C_{\ell_{1}}^{B B} \propto r \propto \epsilon$ (since we are now considering not so small $r$ ). With a fact that $B_{\ell_{1} \ell_{2} \ell_{3}}^{B X X} \propto \epsilon$, we derive $\Delta \lambda_{s s t} \propto \epsilon^{-1 / 2}$. These relations are confirmed from the lines except for $\left(\epsilon, N_{\text {tot }}\right)=\left(10^{-2}, 60\right)$. Because of this, $\Delta \lambda_{\text {sst }}$ at $\ell_{\max }=2000$ depends strongly on $\epsilon$, such as $10^{3}-10^{4}\left(10^{2}-10^{3}\right)$ for $\epsilon=10^{-3}\left(10^{-2}\right)$. Note that our $\Delta \lambda_{\text {sst }}$ for $N_{\text {tot }}=0$ obtained from TTT (TTB) is in agreement with the corresponding results reported in Ref. [56] (Ref. [62]).

It is important to investigate how much the late-time secondary contributions contaminate the primordial signal discussed above. We now consider the TTT, EEE, TTB and EEB bispectra induced by the primordial temperature and E-mode signal via late-time gravitational lensing, which are the dominant components of the secondary contributions. Because of the smallness of the primordial BB spectrum and the absence of the parity-violating primordial correlators such as TB or EB, the leading-order expressions are given by the products of the lensed TT/TE/EE spectrum and the temperature/E-mode polarizationlensing potential cross power spectrum [63,64]. In the bottom panel of Fig. 3 we describe the biases due to such lensed contributions on the estimation of $\lambda_{s s t}$, computed according to $\lambda_{s s t}^{\text {lens }}=F_{\text {sst,lens }} / F_{\text {sst,sst }}$. From the TTT result, we find that $\left|\lambda_{\text {sst }}^{\text {lens }}\right|$ surpasses $\Delta \lambda_{s s t}$ for $\ell_{\max } \gtrsim 1000$. This suggests a caveat against the $\lambda_{\text {sst }}$ estimation without the subtraction of the secondary lensed contributions in the case that one uses the information of TTT beyond $\ell \simeq 1000$. In contrast, there will be no such a concern in the data analysis with EEE, TTB or EEB (at least up to $\ell=2000$ ) because of $\left|\lambda_{\text {sst }}^{\text {lens }}\right| \ll \Delta \lambda_{\text {sst }}$ for all $\ell_{\text {max }}$ 's.

\footnotetext{
${ }^{3}$ The comparable results will be obtained even if one analyzes with the noise spectra expected in other futuristic B-mode surveys such as CMBS4 [28] and COrE [29].
} 

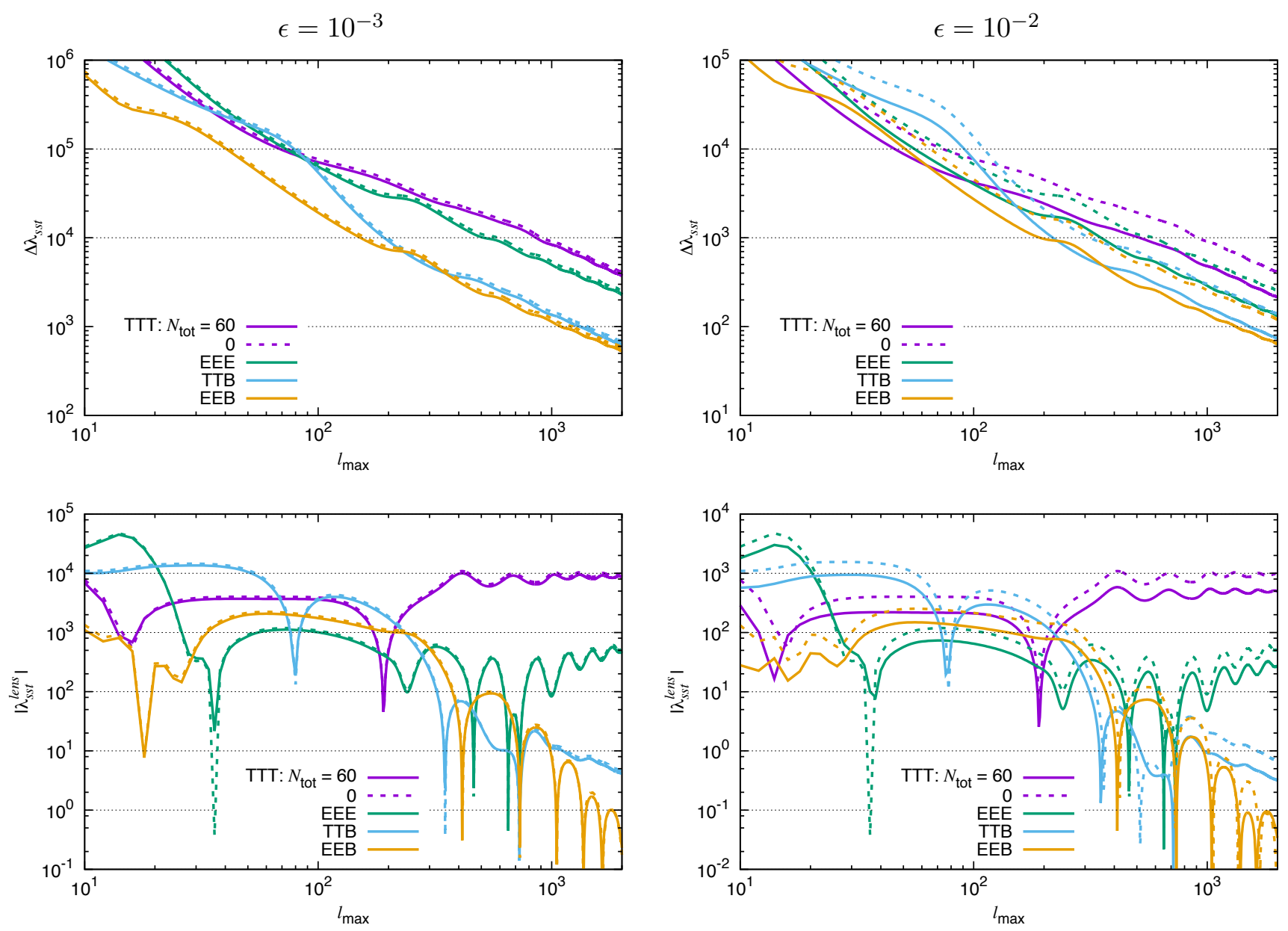

Figure 3. Expected $1 \sigma$ errors on $\lambda_{s s t}$ (top two panels) and the biases due to the secondary lensed bispectra on the estimation of $\lambda_{\text {sst }}$ (bottom two panels) with $\epsilon=10^{-3}$ and $10^{-2}$, computed from the Fisher matrices of TTT, EEE, TTB and EEB. Solid and dotted lines correspond to the cases for $N_{\text {tot }}=60$ and 0 , respectively.

\section{Conclusion and Discussion}

Dynamics of the standard, single-field slow-roll inflation is now well understood including the properties of the linear perturbation, and the theoretical predictions, i.e. the 2-point function or the power spectrum, the spectral index, and the tensor-to-scalar ratio have been tested against the observed CMB data, which resulted in a rather tight constraint on the single-field slow-roll models.

In contrast, the non-linear dynamics is still far from complete understanding. It is expected that it is the key to learn more about the theory of gravity in the early Universe, in particular through the 3-point functions. In this connection, the latest reported Planck results on non-Gaussianities lead us to speculate that actually non-Gaussianities, in particular the local-type ones, might be scale dependent. If this was the case, all models of single-field slow-roll inflation would be excluded because the main contribution to the bispectrum in those models comes from the cubic scalar interaction which must be first of all very small, and which wouldn't produce such scale-dependence. Of course, one may achieve such a feature by resorting to multifield models, with some tuning of the parameters. 
In this work, we proposed an alternative scenario. We proposed a model in which the bispectrum is mainly sourced by the almost scale-invariant primordial scalar-scalar-tensor interactions during inflation. We achieved a sizeable interaction, actually larger than the one from the cubic scalar interaction, by considering a model in which there exists a preferred spatial frame, which breaks the local SO(3) symmetry (part of the local Lorentz symmetry). This is realized by introducing three scalar fields which turn to be non-dynamical and give rise to a preferred spatial frame. It leads to an effective mass for the tensor modes and to an enhancement of the interaction between scalar and tensor modes. We showed that it generates scale-dependent non-Gaussianities similar to what WMAP and Plank probed at low $\ell$. The scale dependence in the CMB bispectrum is naturally obtained because the tensor model transfer function is strongly scale-dependent due to the decay of the tensor mode after it re-enters the horizon.

We parametrized the amplitude of the interaction term and, hence, of the non-Gaussianity with the parameter $\lambda_{s s t}$, Eq.(3.35). We found that $\lambda_{s s t} \epsilon>1$ is required in order to explain the current amplitude at low $\ell$ multipoles. We performed a thorough study of the effects of this 3-point function on the temperature and polarization bispectra. From the Fisher matrix analysis based on an accurate all-sky CMB formalism, we found that, a minimum detectable $\lambda_{\text {sst }}$ from TTT (EEE) up to $\ell=2000$ is $\simeq 4 / \epsilon(2 / \epsilon)$, and it could be improved by more than one order of magnitude if using the information of TTB or EEB. It is therefore interesting to analyze both the current temperature/E-mode maps in WMAP and Planck and the B-mode one in the futuristic CMB experiments with the shape and scale dependence of non-Gaussianity that we have proposed.

\section{Acknowledgement}

This work was supported in part by the MEXT KAKENHI Nos. $15 \mathrm{H} 05888$ and 15K21733. T. Hiramatsu is supported by JSPS Grant-in-Aid for Scientific Research on Innovative Areas No. 16H01098, and No. 15H05888. C. Lin is supported by JSPS postdoc fellowship for overseas researchers, and by JSPS Grant-in-Aid for Scientific Research No .15F15321. M. Shiraishi is supported in part by a Grant-in-Aid for JSPS Research under Grant No. 27-10917, and in part by the World Premier International Research Center Initiative (WPI Initiative), MEXT, Japan. Numerical computations by M. Shiraishi were in part carried out on Cray XC30 at Center for Computational Astrophysics, National Astronomical Observatory of Japan. Y. Wang is supported by the CRF Grants of the Government of the Hong Kong SAR under HKUST4/CRF/13G and ECS 26300316. Y. Wang would like to thank the Yukawa Institute for Theoretical Physics for hospitality where part of this work was done.

\section{A On the gauges}

In this appendix, we give some explicit formulas which might be useful to reproduce the main part of the paper. At the same time, we show that the scalar-scalar-tensor interaction we consider in our model is not a gauge artifact. Form the definition of $Z^{i j}$, equation (2.4), we have that up to the second order in perturbations

$$
Z^{i j}=h^{i j}+h^{(i k} \partial_{k} \pi^{j)}+\partial_{k} \pi^{i} \partial_{k} \pi^{j}-\left(N^{i}+\dot{\pi}^{i}\right)\left(N^{j}+\dot{\pi}^{j}\right) .
$$

It is instructive to see that at the leading order we have

$$
3 \bar{\delta} Z^{i j} \simeq \gamma_{i j}+\partial_{i} \partial_{j}(E-\pi)+O(2),
$$


where we decomposed $\pi^{i}=\pi_{T}^{i}+\partial^{i} \pi$ with $\partial_{i} \pi_{T}^{i}=0$, thus leading to the second order action (3.5). Most importantly, we see that $E$ and $\pi$ appear in a gauge invariant combination [18], that is $E_{\pi}=E-\pi$. The first order constraint yields $E_{\pi}=0$. Let us fix the spatial gauge degrees of freedom to $E=\pi=0$ for simplicity. Thus, up to second order we obtain

$$
3 \bar{\delta} Z^{i j}=\gamma_{i j}-\frac{3}{2} \gamma_{i k} \gamma_{k j}+\frac{1}{6} \delta_{i j} \gamma_{k l} \gamma_{k l}+a^{2} \partial_{i} \beta \partial_{j} \beta-\frac{a^{2}}{3} \delta_{i j} \partial_{k} \beta \partial_{k} \beta+O(3) .
$$

From this expression, it is easy to reproduce the third order action (3.31). Let us recall that in the spatially isotropic comoving slicing gauge one has $[53,65,66]$

$$
\beta_{\text {com }}=-\frac{\mathcal{R}}{H}+a^{2} \epsilon \partial^{-2} \dot{\mathcal{R}}
$$

and in the spatially isotropic flat slicing gauge,

$$
\beta_{\text {flat }}=-\frac{H}{\dot{\phi}} a^{2} \epsilon\left(\partial^{-2} \dot{\delta} \phi+(\epsilon+\delta) H \partial^{-2} \delta \phi\right)
$$

where $\delta=\ddot{\phi} /(H \dot{\phi})$. Regarding the interaction term, once the spatial gauge is fixed, it is given by

$$
\mathcal{L}_{s s t} \supset \bar{\delta} Z^{i j} \partial^{\mu} \varphi^{i} \partial_{\mu} \phi \partial^{\nu} \varphi^{j} \partial_{\nu} \phi=\frac{\dot{\phi}}{3} \gamma_{i j} \partial_{i}(\beta+\delta \phi / \dot{\phi}) \partial_{j}(\beta+\delta \phi / \dot{\phi}) .
$$

In particular, notice that it appears in a temporal gauge invariant combination [66, 67]. This shows the existence of the scalar-scalar-tensor interaction irrespective of the gauge.

\section{References}

[1] R. Brout, F. Englert and E. Gunzig, Annals Phys. 115, 78 (1978).

[2] A. A. Starobinsky, JETP Lett. 30, 682 (1979) [Pisma Zh. Eksp. Teor. Fiz. 30, 719 (1979)].

[3] A. H. Guth, Phys. Rev. D 23, 347 (1981).

[4] K. Sato, Mon. Not. Roy. Astron. Soc. 195, 467 (1981).

[5] C. Lin and L. Z. Labun, "Effective Field Theory of Broken Spatial Diffeomorphisms," JHEP 1603, 128 (2016) [arXiv:1501.07160 [hep-th]].

[6] S. Endlich, A. Nicolis and J. Wang, "Solid Inflation," JCAP 1310, 011 (2013) [arXiv:1210.0569 [hep-th]].

[7] N. Bartolo, S. Matarrese, M. Peloso and A. Ricciardone, "Anisotropy in solid inflation," JCAP 1308, 022 (2013) [arXiv:1306.4160 [astro-ph.CO]].

[8] M. Akhshik, R. Emami, H. Firouzjahi and Y. Wang, "Statistical Anisotropies in Gravitational Waves in Solid Inflation," JCAP 1409, 012 (2014) [arXiv:1405.4179 [astro-ph.CO]].

[9] D. Cannone, G. Tasinato and D. Wands, "Generalised tensor fluctuations and inflation," arXiv:1409.6568 [astro-ph.CO].

[10] Y. Hidaka, T. Noumi and G. Shiu, "Effective field theory for spacetime symmetry breaking," Phys. Rev. D 92, no. 4, 045020 (2015) [arXiv:1412.5601 [hep-th]].

[11] C. Lin and M. Sasaki, "Resonant Primordial Gravitational Waves Amplification," Phys. Lett. B 752, 84 (2016) [arXiv:1504.01373 [astro-ph.CO]]. 
[12] D. Cannone, J. O. Gong and G. Tasinato, "Breaking discrete symmetries in the effective field theory of inflation," JCAP 1508, no. 08, 003 (2015) [arXiv:1505.05773 [hep-th]].

[13] M. Fierz, W. Pauli, Proc. Roy. Soc. Lond. A173, 211-232 (1939).

[14] N. Arkani-Hamed, H. Georgi and M. D. Schwartz, "Effective field theory for massive gravitons and gravity in theory space," Annals Phys. 305, 96 (2003) [hep-th/0210184].

[15] C. de Rham, G. Gabadadze and A. J. Tolley, "Resummation of Massive Gravity," Phys. Rev. Lett. 106, 231101 (2011), [arXiv:1011.1232 [hep-th]].

[16] S. F. Hassan and R. A. Rosen, "Resolving the Ghost Problem in non-Linear Massive Gravity," Phys. Rev. Lett. 108, 041101 (2012), [arXiv:1106.3344 [hep-th]].

[17] A. E. Gumrukcuoglu, C. Lin and S. Mukohyama, "Open FRW universes and self-acceleration from nonlinear massive gravity," JCAP 1111, 030 (2011), [arXiv:1109.3845 [hep-th]].

[18] A. E. Gumrukcuoglu, C. Lin and S. Mukohyama, "Cosmological perturbations of self-accelerating universe in nonlinear massive gravity," JCAP 1203 (2012) 006, [arXiv:1111.4107 [hep-th]].

[19] A. De Felice, A. E. Gmrkolu, C. Lin and S. Mukohyama, "Nonlinear stability of cosmological solutions in massive gravity," JCAP 1305, 035 (2013) [arXiv:1303.4154 [hep-th]].

[20] S. L. Dubovsky, "Phases of massive gravity," JHEP 0410, 076 (2004) [hep-th/0409124].

[21] C. Lin, "SO(3) massive gravity," Phys. Lett. B 727, 31 (2013), [arXiv:1305.2069 [hep-th]].

[22] C. Lin, "Massive Graviton on a Spatial Condensate," Phys. Lett. B 738, 386 (2014) [arXiv:1307.2574 [hep-th]].

[23] D. Comelli, F. Nesti and L. Pilo, "Nonderivative Modified Gravity: a Classification," JCAP 1411, no. 11, 018 (2014) [arXiv:1407.4991 [hep-th]].

[24] A. De Felice and S. Mukohyama, "Minimal theory of massive gravity," Phys. Lett. B 752, 302 (2016) [arXiv:1506.01594 [hep-th]].

[25] C. de Rham, "Massive Gravity," Living Rev. Rel. 17, 7 (2014), [arXiv:1401.4173 [hep-th]].

[26] D. H. Lyth, "What would we learn by detecting a gravitational wave signal in the cosmic microwave background anisotropy?," Phys. Rev. Lett. 78, 1861 (1997). [hep-ph/9606387].

[27] M. Hazumi et al., LiteBIRD: a small satellite for the study of B-mode polarization and inflation from cosmic background radiation detection", Proc. SPIE Int. Soc. Opt. Eng. 8442, no. 844219 (2012).

[28] K. N. Abazajian et al. [CMB-S4 Collaboration], "CMB-S4 Science Book, First Edition," arXiv:1610.02743 [astro-ph.CO].

[29] F. R. Bouchet et al. [COrE Collaboration], "COrE (Cosmic Origins Explorer) A White Paper," arXiv:1102.2181 [astro-ph.CO].

[30] E. Komatsu et al. [WMAP Collaboration], "Seven-Year Wilkinson Microwave Anisotropy Probe (WMAP) Observations: Cosmological Interpretation," Astrophys. J. Suppl. 192, 18 (2011), [arXiv:1001.4538 [astro-ph.CO]].

[31] C. L. Bennett et al. [WMAP Collaboration], Astrophys. J. Suppl. 208, 20 (2013) [arXiv:1212.5225 [astro-ph.CO]].

[32] P. A. R. Ade et al. [Planck Collaboration], Astron. Astrophys. 571, A24 (2014) [arXiv:1303.5084 [astro-ph.CO]].

[33] P. A. R. Ade et al. [Planck Collaboration], "Planck 2015 results. XVII. Constraints on primordial 
non-Gaussianity," arXiv:1502.01592 [astro-ph.CO].

[34] A. D. Linde and V. F. Mukhanov, "Nongaussian isocurvature perturbations from inflation," Phys. Rev. D 56, 535 (1997), [astro-ph/9610219].

[35] M. Sasaki, J. Valiviita and D. Wands, "Non-Gaussianity of the primordial perturbation in the curvaton model," Phys. Rev. D 74, 103003 (2006), [astro-ph/0607627].

[36] G. Dvali, A. Gruzinov and M. Zaldarriaga, "A new mechanism for generating density perturbations from inflation," Phys. Rev. D 69, 023505 (2004), [astro-ph/0303591].

[37] L. Kofman, "Probing string theory with modulated cosmological fluctuations," astro-ph/0303614.

[38] T. Suyama and M. Yamaguchi, "Non-Gaussianity in the modulated reheating scenario," Phys. Rev. D 77, 023505 (2008), [arXiv:0709.2545 [astro-ph]].

[39] X. Chen and Y. Wang, "Large non-Gaussianities with Intermediate Shapes from Quasi-Single Field Inflation," Phys. Rev. D 81, 063511 (2010), [arXiv:0909.0496 [astro-ph.CO]].

[40] X. Chen and Y. Wang, "Quasi-Single Field Inflation and Non-Gaussianities," JCAP 1004, 027 (2010) [arXiv:0911.3380 [hep-th]].

[41] D. Baumann and D. Green, "Signatures of Supersymmetry from the Early Universe," Phys. Rev. D 85, 103520 (2012), [arXiv:1109.0292 [hep-th]].

[42] A. Becker and D. Huterer, Phys. Rev. Lett. 109, 121302 (2012) doi:10.1103/PhysRevLett.109.121302 [arXiv:1207.5788 [astro-ph.CO]].

[43] X. Chen, R. Emami, H. Firouzjahi and Y. Wang, "The TT, TB, EB and BB correlations in anisotropic inflation," JCAP 1408, 027 (2014), [arXiv:1404.4083 [astro-ph.CO]].

[44] L. Bordin, P. Creminelli, M. Mirbabayi and J. Norea, "Tensor Squeezed Limits and the Higuchi Bound," JCAP 1609, no. 09, 041 (2016), [arXiv:1605.08424 [astro-ph.CO]].

[45] R. Namba, M. Peloso, M. Shiraishi, L. Sorbo and C. Unal, "Scale-dependent gravitational waves from a rolling axion," JCAP 1601, no. 01, 041 (2016), [arXiv:1509.07521 [astro-ph.CO]].

[46] C. de Rham, J. T. Deskins, A. J. Tolley and S. Y. Zhou, "Graviton Mass Bounds," arXiv:1606.08462 [astro-ph.CO].

[47] A. Nicolis, R. Rattazzi and E. Trincherini, "The Galileon as a local modification of gravity," Phys. Rev. D 79, 064036 (2009), [arXiv:0811.2197 [hep-th]].

[48] X. Chen and Y. Wang, "Quasi-Single Field Inflation with Large Mass," JCAP 1209, 021 (2012), [arXiv:1205.0160 [hep-th]].

[49] S. Pi and M. Sasaki, "Curvature Perturbation Spectrum in Two-field Inflation with a Turning Trajectory," JCAP 1210, 051 (2012), [arXiv:1205.0161 [hep-th]].

[50] J. O. Gong, S. Pi and M. Sasaki, "Equilateral non-Gaussianity from heavy fields," JCAP 1311, 043 (2013), [arXiv:1306.3691 [hep-th]].

[51] X. Chen, M. H. Namjoo and Y. Wang, "Quantum Primordial Standard Clocks," JCAP 1602, no. 02, 013 (2016), [arXiv:1509.03930 [astro-ph.CO]].

[52] Y. Wang and W. Xue, "Inflation and Alternatives with Blue Tensor Spectra," JCAP 1410, no. 10, 075 (2014), [arXiv:1403.5817 [astro-ph.CO]].

[53] J. M. Maldacena, "Non-Gaussian features of primordial fluctuations in single field inflationary models," JHEP 0305, 013 (2003), [astro-ph/0210603]. 
[54] N. Bartolo, D. Cannone, A. Ricciardone and G. Tasinato, JCAP 1603, no. 03, 044 (2016) doi:10.1088/1475-7516/2016/03/044 [arXiv:1511.07414 [astro-ph.CO]].

[55] Y. Wang, "Inflation, Cosmic Perturbations and Non-Gaussianities," Commun. Theor. Phys. 62, 109 (2014), [arXiv:1303.1523 [hep-th]].

[56] M. Shiraishi, D. Nitta, S. Yokoyama, K. Ichiki and K. Takahashi, "CMB Bispectrum from Primordial Scalar, Vector and Tensor non-Gaussianities," Prog. Theor. Phys. 125 (2011) 795, [arXiv:1012.1079 [astro-ph.CO]].

[57] M. Shiraishi, S. Yokoyama, K. Ichiki and K. Takahashi, "Analytic formulae of the CMB bispectra generated from non-Gaussianity in the tensor and vector perturbations," Phys. Rev. D 82, 103505 (2010), [arXiv:1003.2096 [astro-ph.CO]].

[58] E. Komatsu and D. N. Spergel, "Acoustic signatures in the primary microwave background bispectrum," Phys. Rev. D 63 (2001) 063002, [astro-ph/0005036].

[59] T. Matsumura et al., "Mission design of LiteBIRD," J. Low. Temp. Phys. 176, 733 (2014), [arXiv:1311.2847 [astro-ph.IM]].

[60] T. Matsumura et al., "LiteBIRD: Mission Overview and Focal Plane Layout," J. Low. Temp. Phys. 184, no. 3-4, 824 (2016).

[61] J. R. Pritchard and M. Kamionkowski, "Cosmic microwave background fluctuations from gravitational waves: An Analytic approach," Annals Phys. 318, 2 (2005), [astro-ph/0412581].

[62] P. D. Meerburg, J. Meyers, A. van Engelen and Y. Ali-Hamoud, "CMB B -mode non-Gaussianity," Phys. Rev. D 93, 123511 (2016), [arXiv:1603.02243 [astro-ph.CO]].

[63] W. Hu, "Weak lensing of the CMB: A harmonic approach," Phys. Rev. D 62, 043007 (2000), [astro-ph/0001303].

[64] A. Lewis, A. Challinor and D. Hanson, "The shape of the CMB lensing bispectrum," JCAP 1103, 018 (2011), [arXiv:1101.2234 [astro-ph.CO]].

[65] K. Koyama, "Non-Gaussianity of quantum fields during inflation," Class. Quant. Grav. 27 (2010) 124001, [arXiv:1002.0600 [hep-th]].

[66] H. Kodama and M. Sasaki, "Cosmological Perturbation Theory," Prog. Theor. Phys. Suppl. 78 (1984) 1.

[67] V. F. Mukhanov, H. A. Feldman and R. H. Brandenberger, "Theory of cosmological perturbations. Part 1. Classical perturbations. Part 2. Quantum theory of perturbations. Part 3. Extensions," Phys. Rept. 215 (1992) 203. 